\title{
Transbronchial needle aspiration in clinical practice
}

\author{
EDWARD M HARROW, FREDERICK A OLDENBURG, A MARSHALL SMITH
}

From the Department of Medicine, Eastern Maine Medical Center, and St Joseph Hospital, Bangor, Maine, USA

ABSTRACT Transbronchial needle aspiration has recently been adapted for use with the flexible bronchoscope. We studied 108 patients, who had a total of 110 aspirations performed, and diagnosed thoracic cancer in 70 cases. Transbronchial needle aspiration revealed malignant disease in $32(46 \%)$ of these 70 patients. In $12(17 \%)$ patients with cancer this technique provided the sole cytological or histological confirmation of the diagnosis. It gave a positive result in an additional $20(29 \%)$ cases in which the diagnosis was also proved by conventional procedures, and it provided important staging information in these patients. In $20 \%$ of patients with cancer transbronchial needle aspiration precluded the need for further diagnostic surgery. There were no complications in this series. This is a safe, useful, and economical technique that can be used in diagnosing and staging patients with carcinoma of the lung.

The introduction of transbronchial needle aspiration has enabled bronchoscopists to sample tissue beyond the confines of the tracheobronchial tree. This technique, which was first undertaken with the rigid bronchoscope,' has been modified recently for application with the flexible instrument. Much of this experience has involved its use in sampling primarily intraluminal lesions under direct vision that can also be approached with a biopsy forceps or brush. $^{2-4}$ Wang and Terry ${ }^{5}$ have extended the capabilities of this method by obtaining aspirates from the mediastinal nodes adjacent to the trachea in an attempt to stage bronchogenic carcinoma.

The primary advantage of this technique appears to lie in its ability to provide important diagnostic and staging information from extraluminal sites in the evaluation of patients with lung cancer at low cost and with minimal risk by comparison with mediastinoscopy or thoracotomy. Previous reports have described the use of this technique in academic practice. We described our experience with transbronchial needle aspiration in over 100 patients in a community hospital setting (where most patients receive their medical care) and assess its utility, economy, and safety.

\section{Methods}

This study described transbronchial needle aspiration in 108 consecutive patients in whom we per-

Address for reprint requests: Dr Edward M Harrow, 417 State Street, Bangor, Maine 04401, USA.

Accepted 7 May 1985 formed bronchoscopy for suspected carcinoma at Eastern Maine Medical Center (425 beds) and St Joseph Hospital (130 beds) in Bangor, Maine, from May 1983 to April 1984. As respirologists, we serve the 350000 people of north eastern Maine. Those who were suspected of having lung cancer according to the usual clinical criteria had transbronchial needle aspiration performed as an integral part of their bronchoscopic evaluation. A transoral approach through an endotracheal tube was used for the flexible fibreoptic bronchoscopy.

Transbronchial needle aspiration was performed with a needle that consists of a $120 \mathrm{~cm}$ long hollow semitranslucent polyethylene sheath tipped with a 22 gauge needle at one end and a sideport at the other (Mill Rose Co, Mentor, Ohio). An inner steel stylette extends through its entire length and protrudes beyond the needle tip. After the bronchoscope has been passed into the trachea and a brief examination of the proximal portion of the tracheobronchial tree has been accomplished, the instrument is positioned in the distal trachea and the needle is passed through the bronchoscopic channel until it is visualised in the tracheal lumen. The stylette is then withdrawn into the needle in order to maintain sufficient rigidity between the needlesheath interface to permit puncture of the tracheal wall. This is accomplished by a quick thrust between the tracheal rings in infracarinal and paratracheal positions adjacent to the nodes to be sampled. The stylette is further withdrawn into the sheath and the needle vibrated by a rapid to and fro motion at the distal end of the sheath while material is aspirated with maximally applied suction into a $50 \mathrm{ml}$ syringe 
containing $3-5 \mathrm{ml}$ of saline. The suction is then released before the needle is removed from its position so that the sample is not contaminated by intraluminal secretions. Two or three aspirations from each position are obtained and flushed through the needle into an appropriate container for cytological examination. After collection of these samples the formal bronchoscopic examination is performed.

Patients were studied both as inpatients and as outpatients, but transbronchial needle aspiration was never considered a necessary precondition for hospital admission. During clinical evaluation the diagnosis of cancer was made by various procedures, including percutaneous needle aspiration, direct bronchial or transbronchial lung biopsy and brushing, mediastinoscopy, and thoracotomy as well as transbronchial needle aspiration. The diagnosis of metastatic carcinoma was made on clinical grounds alone in three patients, who had a compatible chest radiograph and a tissue diagnosis obtained from another organ.

Radiographic studies other than chest radiography were performed only when thought to be clinically indicated. Cytological analysis was performed by standard techniques and findings were interpreted as positive only when large numbers of unequivocally malignant cells were obtained. Cytological specimens reported as suspicious were considered negative.

\section{Results}

The first 110 transbronchial needle aspirations serve as the basis for this analysis. Two patients had the study performed twice: in one case to demonstrate the recurrence of tumour after treatment and in the other to establish a diagnosis after an initially negative result from the aspiration. In this latter case computed tomography of the chest confirmed the presence of abnormal nodes in the aortopulmonary window and the repeat aspiration gave a positive result.

The diagnosis of cancer was established by at least one method in 70 patients. Transbronchial needle aspiration showed malignant cells in eight of 11 cases of small cell carcinoma, 10 of 17 cases of large cell carcinoma, seven of 18 cases of adenocarcinoma, and six of 20 cases of squamous cell carcinoma. One patient who was shown to have lymphoma by transbronchial needle aspiration had the diagnosis confirmed by an exploratory thoracotomy. Three putients with metastatic disease of the lungs had a negative result from the aspiration, as did two with carcinoid tumours.

Transbronchial needle aspiration performed at sites of N2 nodes (paratracheal or subcarinal) gave a positive result in 19 of 44 cases $(43 \%)$ of right sided tumour and six of 22 cases $(27 \%)$ of left sided tumour. The patient with lymphoma had a posterior mediastinal mass.

In nine of the cases in which malignancy was diagnosed transbronchial needle aspiration gave a positive result in more peripheral areas of the tracheobronchial tree. Three of these nine also had positive aspirates at $\mathrm{N} 2$ sites and six of them had positive biopsy specimens. In two cases peripheral positive aspirates provided the sole pathological evidence confirming the diagnosis of cancer.

In 20 cases, transbronchial needle aspiration and bronchial biopsy both established the diagnosis of malignant disease. There was generally close agreement between samples in respect of the cell type. Discrepant results were, however, noted in two cases. In one the biopsy specimen was reported to show large cell carcinoma while the aspirate was reported as positive for small cell carcinoma. In the second case the reverse was true. In each case the tumour was classified in accordance with the biopsy interpretation.

Our results are summarised in the table. In patients who were proved to have lung cancer, transbronchial needle aspiration was the sole means of demonstrating malignant cells and therefore confirmed the diagnosis in $17 \%$ (12 of 70 ) cases. Positive results were obtained in an additional $29 \%$ ( 20 of 70) where another technique also confirmed the diagnosis of malignancy and in these cases it provided additional staging information. It obviated the need for mediastinoscopy in 11 patients who would otherwise have been candidates for this procedure, either to prove the presence of malignancy or to determine operability. One additional patient was spared a thoracotomy because of a positive result from transbronchial needle aspiration. He had undergone a right upper lobectomy with postoperative radiotherapy three years previously, but subsequently developed subcarinal lymphadenopathy, which would have been difficult to approach by standard mediastinoscopy. Finally, a repeat bron-

Results of transbronchial needle aspiration (TBNA) in 108 patients

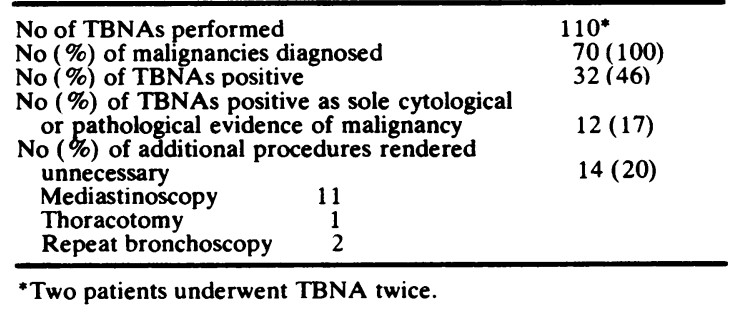


choscopy was avoided in two additional cases when transbronchial needle aspiration gave a positive result although biopsy specimens of visible tumour were negative.

Of 26 patients with positive results from transbronchial needle aspiration of $\mathrm{N} 2$ nodes (25 carcinoma and one lymphoma), 14 cases $(54 \%)$ had radiographic features of mediastinal lymphadenopathy. More interestingly, this technique also demonstrated malignant cells in 12 cases (46\%) in which the chest radiograph did not indicate the presence of mediastinal disease. Aspirates of N2 sites in patients with cancer were positive in 16 patients $(62 \%)$ when extrinsic compression or distortion of the trachea was evident, but were also positive in 10 individuals $(38 \%)$ when the distal trachea appeared unremarkable.

Twenty three patients with cancer had chest radiographs that showed mediastinal lymphadenopathy and in $14(61 \%)$ of these transbronchial needle aspiration yielded malignant cells. One other patient had a negative result from mediastinoscopy. Of the remaining eight, five were thought to have mediastinal disease on clinical grounds (paralysed vocal cord, paralysed diaphragm, pericarditis, positive oesophageal cytological appearances, positive supraclavicular nodes) and another was shown to have enlarged nodes by computed tomography. The state of the remaining two patients was uncertain.

Thirty eight of the patients with cancer had a negative result from transbronchial needle aspiration at sites of $\mathrm{N} 2$ nodes. Fifteen of these patients underwent mediastinoscopy and in 12 the lymph nodes were free from tumour while only three showed the presence of tumour.

Although the aspiration of blood occurred about $25 \%$ of the time, this did not require a change in standard postoperative care. No other complications were encountered, although after the first 25 procedures postoperative chest radiographs were not performed routinely.

\section{Discussion}

The technique of transbronchial needle aspiration has been available to bronchoscopists since 1958.' Its modification for the fibreoptic instrument has considerably expanded its use..$^{2-5}$ Our study describes the application of this technique in our clinical practice and shows its value. Transbronchial needle aspiration gave a positive result in $29 \%$ of cases in which the diagnosis of carcinoma of the lung was considered. It provided important staging information (positive $\mathrm{N} 2$ nodes) in 26 cases or $37 \%$ of patients in whom cancer was diagnosed and was the sole histological means by which this diagnosis was confirmed in $17 \%$ of cases. It precluded the need for additional and usually more extensive surgical procedures (mediastinoscopy, thoracotomy, or repeat bronchoscopy) for diagnosis or staging in $20 \%$ of our patients with malignant disease. Our finding of positive results from transbronchial needle aspiration in $29 \%$ of all cases and $46 \%$ of patients shown to have carcinoma is significantly lower than the $49 \%$ and $64 \%$ that have been reported. ${ }^{6}$ Differences in case selection may help to explain this disparity, since carcinoma had already been diagnosed before the procedure in nearly $30 \%$ of the patients in the earlier series.

Since ours is a community practice, patients were managed strictly on clinical grounds and computed tomography and mediastinoscopy were not performed routinely. As a result, sensitivity and specificity of transbronchial needle aspiration in this study have not been determined. Nevertheless, three of 15 cases $(20 \%)$ with negative results from transbronchial needle aspiration who were surgical candidates were shown on mediastinal exploration to have malignant disease of $\mathrm{N} 2$ nodes. Five others were thought to have similar tumour extension on clinical grounds. This technique therefore cannot be used to exclude neoplastic mediastinal invasion.

Three of our cases had sarcoidosis proved by mediastinal biopsy but transbronchial needle aspiration was unsuccessful in establishing the diagnosis. The recent study by Pauli et al, ${ }^{7}$ in which a $1.5 \mathrm{~mm}$ diameter needle was used successfully in $66 \%$ of cases where the aspiration was performed through a rigid bronchoscopy, suggests that the 22 gauge needle we used with the flexible bronchoscope is not large enough for diagnosing non-malignant diseases.

Interpretation of cytological specimens has not been difficult. Positive specimens are nearly always unequivocal and show large numbers of frankly malignant cells. We do not accept the presence of rare malignant cells as being indicative of a positive aspiration biopsy specimen, and would in fact suspect contamination of the aspirate by intraluminal tumour if such a report were received. This type of case was recently discussed by Cropp et al. ${ }^{8}$ Suspicious cytological findings should also be considered negative, although in our two cases carcinoma was confirmed by a different technique. Adherence to these criteria as well as careful bronchoscopic technique should make the likelihood of false positive interpretation of transbronchial needle aspirates remote.

In two of 20 cases in which both transbronchial needle aspiration and biopsy specimens were positive for malignancy different interpretations of tumour type (small versus large cell carcinoma) 
were made. Since the treatment for non-resectable tumours may be quite different, the potential for inaccurate determination of cell type in patients who have the diagnosis of cancer made exclusively by transbronchial needle aspiration should be carefully considered before treatment is initiated.

There have been no serious complications, although the aspiration of blood is not uncommon. In these cases the needle is simply removed and repositioned several millimetres from the site where blood had been aspirated, and the procedure is repeated. Accordingly, we do not routinely perform chest radiographs postoperatively and do not consider that rigid bronchoscopy need be immediately available, as has also been suggested. ${ }^{5}$

Our practice has been to perform transbronchial needle aspiration on a routine basis on patients undergoing bronchoscopy when the diagnosis of carcinoma of the lung is a possibility. By using this approach definitive information for diagnosis and staging can be obtained at the time of initial bronchoscopy and the need for a repeat bronchoscopic examination for diagnostic or staging purposes or a more invasive surgical procedure may be obviated. The fact that transbronchial needle aspirates of the distal trachea in patients with lung cancer were positive $46 \%$ of the time when chest radiographs did not show mediastinal disease and $38 \%$ of the time when the anatomy appeared unremarkable provides support for this policy.

We consider patients inoperable when transbronchial needle aspirates are positive in $\mathrm{N} 2$ nodes and plain radiographs, tomograms, or computed tomography show mediastinal adenopathy. The meaning of a positive specimen from $\mathrm{N} 2$ sites in the absence of radiologically demonstrable nodes by computed tomography remains unclear. Naruke et al $^{9}$ report that subcarinal adenopathy indicates a more ominous prognosis than does paratracheal disease. This is presumed to be due to the increased incidence of malignant contralateral nodes when subcarinal nodes bear tumour. Our management of these patients depends on their overall medical state. Many of our patients are elderly or have significant coexistent pulmonary, cardiac, or vascular disease. Although any one or a combination of these conditions does not preclude operatibility, we believe that their coexistence along with carinal or paratracheal aspirates showing many malignant cells are usually sufficient to exclude them from surgical treatment.
Finally, the cost savings of this procedure are substantial. At our institutions outpatient bronchoscopy with transbronchial needle aspiration is about one third of the cost of a standard mediastinoscopy. If the aspiration is performed as part of the initial bronchoscopic procedure rather than as a staging technique in patients with suspected pulmonary malignancy, then the entire cost of further diagnostic surgery can be saved in many of these cases.

In summary, we have found transbronchial needle aspiration to be a safe, economical, and useful technique in the diagnosis and assessment of patients with lung cancer.

We are indebted to Dr Ko-Pen Wang, who instructed us in the application of this technique, encouraged the publication of this series, and reviewed this manuscipt. We also wish to thank Ms Renee Melanson for assistance in its preparation and Ms Freda Davis, Jill DeCesere, and Gail Merrow for help with the data.

\section{References}

1 Schieppati E. Mediastinal lymph node puncture through the tracheal carina. Surg Gyn Obstet 1958; 110: 243-6.

2 Oho K, Kato H, Ogawa I, Hayashi N, Hayata Y. A new needle for trans-fiberoptic bronchoscopic use. Chest 1979;76:492.

3 Buirski G, Calverly PMA, Douglas NJ, et al. Bronchial needle aspiration in the diagnosis of bronchial carcinoma. Thorax 1981;36:508-11.

4 Lundgren R. A flexible thin needle for transbronchial aspiration biopsy through the flexible fiberoptic bronchoscope. Endoscopy 1980;12:180-2.

5 Wang KP, Terry PB. Transbronchial needle aspiration in the diagnosis and staging of bronchogenic carcinoma. Am Rev Respir Dis 1983;127:344-7.

6 Wang KP, Brower R, Haponik EK, Siegelman S. Flexible transbronchial needle aspiration for staging of bronchogenic carcinoma. Chest 1983;84:571-6.

7 Pauli G, Pelletier A, Bohner C, Roeslin N, et al. Transbronchial needle aspiration in the diagnosis of sarcoidosis. Chest 1984;85:482-4.

8 Cropp A, DiMarco A, Lankerani M. False positive transbronchial needle aspiration in bronchogenic carcinoma. Chest 1984;85:696-7.

9 Naruke T, Suemasu K, Ishikawa S. Lymph node mapping and curability at various levels of metastasis in resected lung cancer. J Thorac Cardiovasc Surg 1978; 76:832. 